\title{
Impact of humic acids on EYL liposome membranes: ESR method
}

\author{
Barbara Pytel, \\ Aneta Filipiak, \\ Izabella Pisarek, \\ Ryszard Olchawa, \\ Dariusz Man
}

\begin{abstract}
In this paper, the effects of model (commercial) and natural (extracted from peat) humic substances on the membrane of liposomes formed with egg yolk lecithin (EYL) are presented. In our research, mass concentrations of fulvic and humic acids were used, which in relation to lecithin varied from $0 \%$ to $13 \%$. To study membrane fluidity, electron spin resonance (EPR) was used with two spin probes, penetrating various regions of the lipid bilayer. The effects of model and natural humic substances (humic acids - HAs and fulvic acids FAs) on the lipid membrane in different regions were researched: the lipid-water interphase, and in the middle of the lipid bilayer. It was shown that FA and HA impact the fluidity of liposome membranes in different ways. Increased mass concentrations of HAs decreased membrane fluidity in both acids: extracted from peat and the model. However, increased mass concentration of FAs extracted from peat, decreased membrane fluidity in the surface region, at the same time stiffening the central part of the bilayer. Increasing the concentration of FAs extracted from peat had the opposite effect when compared to model FA. This effect may be related to the complexation of xenobiotics present in the soil environment and their impact on biological membranes.
\end{abstract}

Key words: EYL liposomes • ESR method • humic substances

B. Pytel, R. Olchawa, D. Man ${ }^{\bowtie}$

Institute of Physics,

Opole University,

48 Oleska Str., 45-052 Opole, Poland,

Tel.: +48 77452 7250, Fax: +48 77452 7290,

E-mail: dariusz.man@uni.opole.pl

A. Filipiak, I. Pisarek

Department of Land Protection,

Opole University,

22 Oleska Str., 45-052 Opole, Poland

Received: 1 October 2014

Accepted: 30 January 2015

\section{Introduction}

Natural humic substances (HS) are heterogeneous mixtures, which result from a variety of organic compounds that have been introduced into the environment and undergone humification by soil microbes and physicochemical factors.

The effect of the process of humification in soil depends on the chemical composition of organic residues, the number and diversity of microorganisms, humidity, temperature, and $\mathrm{pH}$, as well as other factors.

The chemical structure of HS has been the subject of numerous scientific papers for over 200 years. According to Schnitzer [1], it is the main topic of research on HS. In light of previous studies [1-3], it is believed that the humic acid (HA) molecule is a polymer composed of aromatic rings connected by -O-, -NH-, - $\left(\mathrm{CH}_{2}\right) n$, and containing various functional groups (carboxyl, phenol, methoxy, methyl, etc.). In the structural molecules of HS, there are differences in their molecular weights and in the total content of functional groups. The molecular weight of HS varies from a few hundred to several million (from 300 to >2 000 000).

Despite much worldwide research using state-of-the-art analytical methods, a model of the molecular structure of HA is very difficult to construct. This 


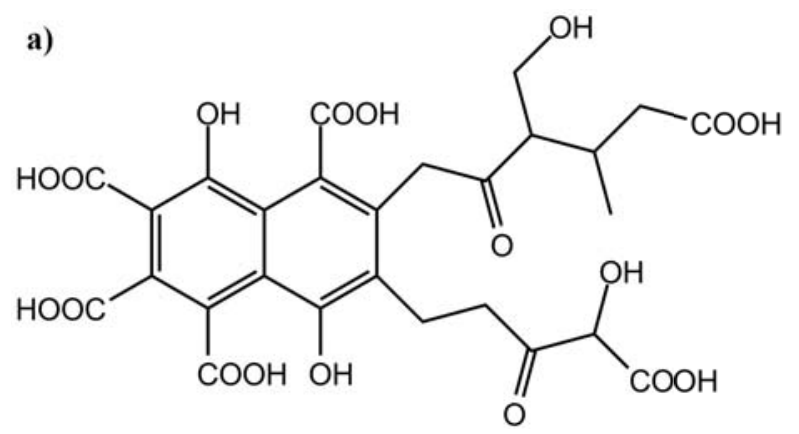

b)

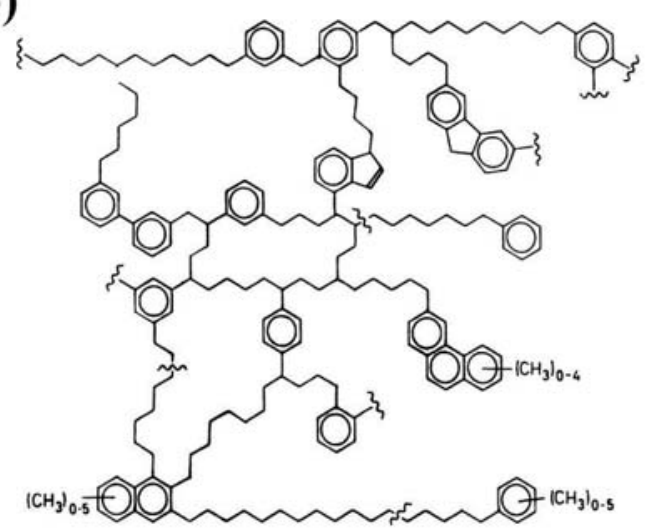

Fig. 1. (a) The structure of fulvic acid proposed by Buffle [4]; (b) the structure of humic acid proposed by Shulten [5].

is due to the fact that in the process of isolating HS from soil, external organic matter, or water, the material is determined by the type of extraction solvent used. Chemical treatment for purification causes structural changes. Over the past few years, many models of the structure of HS have been proposed [1-6]. The structure of these exceptionally complex compounds (Fig. 1) is not yet fully understood, therefore, differences between them are usually compared. Among natural HS, HAs, FAs, and humin can be distinguished.

HS are defined on the basis of differences in solubility. FAs dissolve in water, acid, and alkali. HAs, soluble only in alkaline solutions, are precipitated in the form of gels in an acidic reagent. Humin remains as the residuum. HS are macromolecules formed by microbial catabolism of plants and polymerization of smaller molecules [1]. The chemistry of HS is influenced by functional group heterogeneity and variations in molecular size. The HA and FA, the most common fractions in which the HS are fractionated, have been shown to contain many oxygen-containing groups: carboxyls, hydroxyls, and carbonyls [3]. The molecular weights of HS vary from a few hundred to several millions and among others decides on these mobility. Since parameters of the soil are some of the best indicators of the kind of environment, information 'contained in the humus' can show the state of a particular ecosystem over the years. The habitat in which HS are formed has a direct impact on their internal structure, known as the degree of aromaticity and alifaticity, which in turn determines their mobility and complexation with xenobiotics and consequently their impact on the biosphere [7].
Some research showed $[3,6,8]$ that HS extracted from natural sources could be contaminated by different metal ions. Therefore, ESR spectra, analyzed by wide sweep range could be showed it. In this paper, the impact of HA and FA on the fluidity of biological membranes was presented. Chemical structures of HS may modify properties of membranes by penetration into.

HS are also known to be reach in stable free radicals, which play important roles in different reactions with other organic molecules. At the same time, it has been shown [7, 9-14] that various types of chemical compounds, known as modifiers (including HS), change the physical parameters of biological membranes. There is a relationship between the biological activity modifier (e.g., toxicity affecting living organisms) and its effect on a membrane fluidity model $[8,9]$. This effect is clearly dependent on the concentration of the substance penetrating the lipid bilayer.

The quantitative ESR method was also applied to the investigations of free radicals in HS and was applied in monitoring the impact on biological membranes.

The aim of this study was to determine the effect of different doses of fulvic and humic acids extracted from natural sources (Histosols [15]) and their synthetic counterparts on the fluidity of liposomes' membranes. ESR was used.

\section{Materials and methods}

\section{Humic substances}

Peat, an organic soil, was taken from the surface horizons of Histosols [15]. Model forms of HS, containing $30-40 \%$ of HA was made by Carl Roth $\mathrm{GmbH}+\mathrm{Co}$. KG. HA and FA extractions and purifications were carried out by the Schnitzer method [1] as follows: HS were isolated by the extraction of $10 \mathrm{~g}$ of peat (and respectively $1 \mathrm{~g}$ of model forms of HS) with an aqueous solution of $\mathrm{NaOH} 0.5$ mole followed by centrifuging at room temperature and precipitating at $\mathrm{pH}<1.5$. HAs were separated from FAs by centrifugation, washed with distilled water in order to get a neutral $\mathrm{pH}$, shaken with a mixture of $\mathrm{HF}$ and $\mathrm{HCl}$ at room temperature for $24 \mathrm{~h}$, washed again with distilled water to a neutral $\mathrm{pH}$. The same procedure with FAs (at acidity reaction) was done. The method for isolation of HS from peat with aqueous $\mathrm{NaOH}$, followed by precipitation of $\mathrm{HA}$ at low $\mathrm{pH}$ and a series of desalting steps involving cation exchange, dialysis to the neutral $\mathrm{pH}$ to obtain FA. Through these procedures, all organic acids that are extracted from a solid organic materials are ultimately found in either the HA or FA. Neutral pH is need by using spin probes - alkaline and acidity reaction destroyed these. These methods are generally successful and yield comparable results. Method of extraction of HS [1] was recommended by the International Humic Substance Society (IHSS) (the procedures used by the IHSS to isolate standard and reference HA and FA are simply) as an accept- 

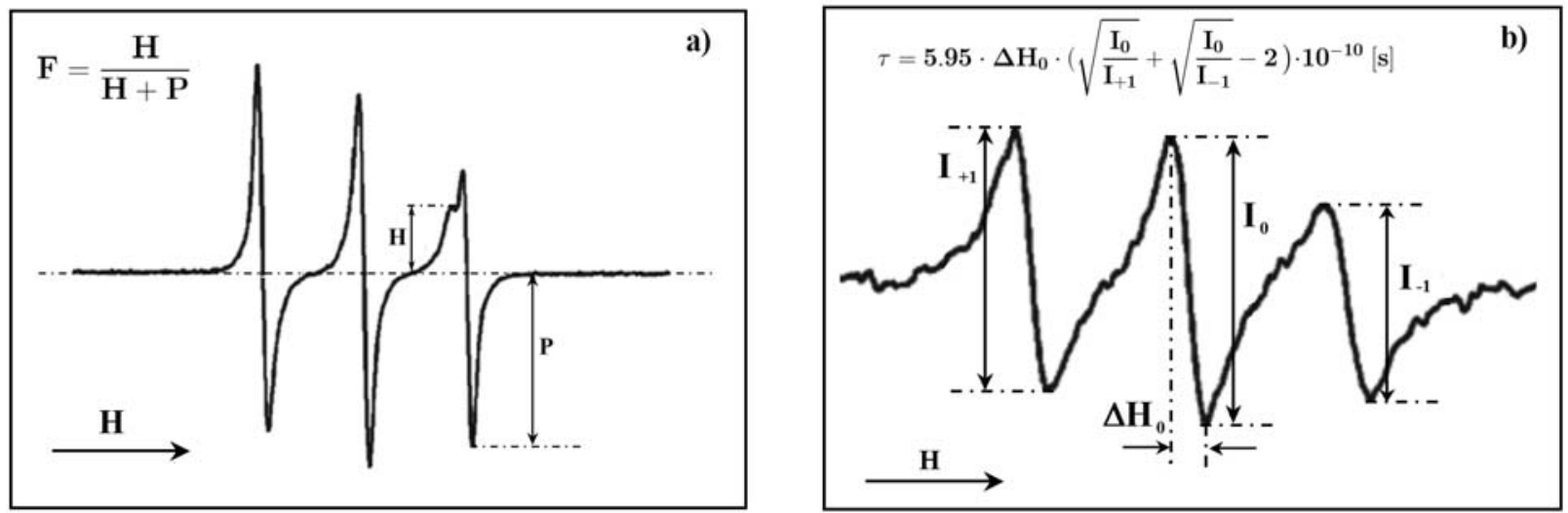

Fig. 2. ESR spectra of the spin probe placed in an aqueous dispersion of liposomes EYL (a) TEMPO, (b) 16-DOXYL.

able method for the extraction of HS from soils and organic materials.

The content of organic carbon in the solutions of HS used was determined by Analyzer multi N/C 3100 (Analytical Jena, Germany, 2013). The HA and FA contents were respectively: HA of peat $2951 \mathrm{mg} / \mathrm{L}$, the model forms of HA's $1722 \mathrm{mg} / \mathrm{L}$, FA of peat: $743.6 \mathrm{mg} / \mathrm{L}$, the model forms of the FA's of $548.8 \mathrm{mg} / \mathrm{L}$

All analyses were performed in three replicates. The samples of HA and FA were extracted as triplicate, and each samples of the ESR measurement was done. The results presented in as a numerous are the arithmetic means.

\section{Liposomes preparation}

Liposomes are formed during the process of egg yolk lecithin (EYL) sonication in an aqueous medium. Lecithin was processed at the Institute of Chemistry, Opole University, as described by Man et al. [13]. A single sample contained $40 \mu \mathrm{M}$ of lecithin in $1 \mathrm{~cm}^{3}$ of distilled water. Sonication was carried out using an ultrasonic disintegrator (TECHPAN UD-20) in six alternate cycles: $30 \mathrm{~s}$ of sonication and $20 \mathrm{~s}$ of cooling (at quartz tube). For the aqueous dispersion of liposomes, a suitable spin probe was introduced, at a concentration of $1000 \mathrm{ppm}$ in relation to the lecithin. In the experiment, there were two spin probes: 2,2,6,6-tetramethylpiperidine-1-oxylane (TEMPO) and 2-ethyl-2-(15-methoxy-15-oxopentadecyl)-4,4-dimethyl-3-oxazolidinyloxy (16-DOXYL stearic acid), which penetrate various parts of the membrane. Weighed samples of HS (prepared from their solutions, after evaporation of the solvent) were introduced into the aqueous dispersion of the liposomes. Due to the complex structure of the HS, concentrations by weight were used, which in relation to lecithin varied from 0 to $13 \%$. In order to thoroughly mix the ingredients, each of the samples was shaken $600 \mathrm{~s}$ (using microshaker with polypropylene tubes) before measuring and then placed in a measuring chamber spectrometer (in glass capillaries of $1 \mathrm{~mm}$ diameter and capacity approx. $0.04 \mathrm{ml}$ ). The ESR measurements were performed at a constant temperature of $22^{\circ} \mathrm{C}$, and the total time of the measurement series was no more than $4 \mathrm{~h}$ for each sample. ESR spectrometer operating parameters were: microwave power $P=$ $60 \mathrm{~mW}$, sweep range $\Delta H=7 \mathrm{mT}$, amplitude modulation $d H=0.08 \mathrm{mT}$, time constant $t=0.3 \mathrm{~s}$, sweep time $t=128 \mathrm{~s}$.

Spin probes were chosen in such a way as to give information from different areas of the liposome membrane. The TEMPO probe was located in both the hydrophobic part of the membrane and in the aquatic medium, providing information about changes in the lipid-water interphase. On the basis of the ESR spectrum obtained by means of TEMPO Fig. 2a, the spectroscopic parameter of partition $(F)$ of this probe in the membrane and its environs was determined. $F$ is defined as the ratio of the relationship of the high-field amplitude lines in the ESR spectrum of the probe dissolved in an aqueous medium $(P)$ to the sum of $P$ and the low-field amplitude of the line originating from the lipid medium $(H)$. The value of $F=H /(H+P)$ is connected, among others, with the fluidity of the membrane [14]. Based on the spectra of the 16-DOXYL probe (Fig. 2b), index $\tau$ (rotational correlation time) was determined. The value of this parameter depends on the fluidity of the lipid bilayer [16-18]. A decrease of parameter $\tau$ indicates an increased interior membrane fluidity - increased rotational speed of the probe.

The spectroscopic parameters $(F$ and $\tau$ ) give information on the dynamic properties of the liposomal membranes being tested, which are affected by the changing concentrations of synthetic and natural humic compounds. The relative measurement errors were set at 2\% for parameter $F$ and 3\% for parameter $\tau$.

\section{Results and discussion}

The liposomes used in our study were in the form of bubbles surrounded by a lipid bilayer membrane composed of phospholipids which surround the chain - proteins called apolipoproteins $[11,12,16$, 17]. The film of liposomes is constructed analogously to these biological membranes. These structures, formed from natural ingredients, are also the same in all cell membranes. Therefore, they can be used as a model in biological, physical, and chemical studies. The membranes were marked by spin probes and 
a)

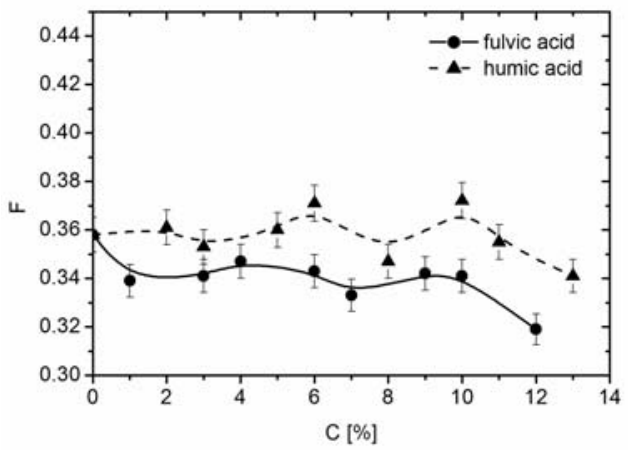

b)

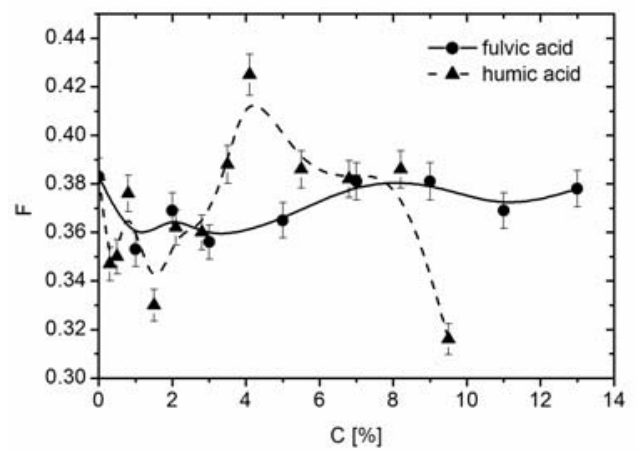

Fig. 3. Changes of spectroscopic parameters $F$ for the probe TEMPO as a function of admixture concentrations of humic substances: (a) natural, (b) synthetic.

a)

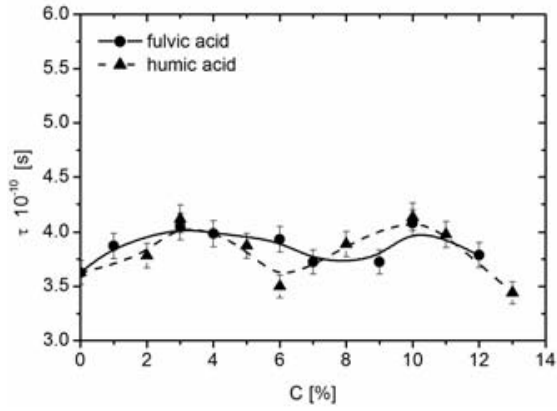

b)

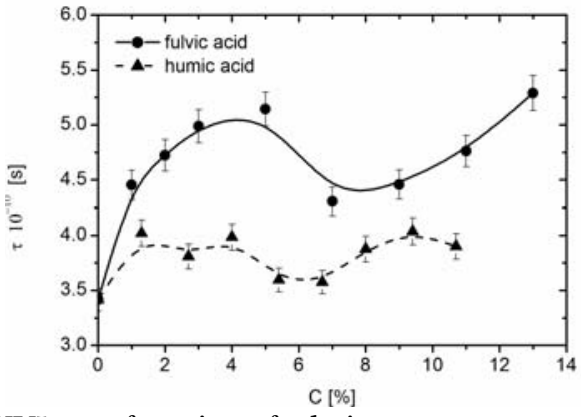

Fig. 4. Changes in the rotational correlation time $\tau$ for probe 16-DOXYL as a function of admixture concentrations of humic substances: (a) natural, (b) synthetic.

under HS, were treated. Changes in the ESR signal coming from the probes were recorded in the range $7 \mathrm{mT}$ - around $g=2$.

Figures 3 and 4 show the effect of admixtures of HA and FA on spectroscopic parameters of spin probes located in the membranes of liposomes. Figs. 3a and 4a illustrate the impact of both acids extracted from peat, while Figs. $3 \mathrm{~b}$ and $4 \mathrm{~b}$ describe the impact of model acids.

This interaction was described by changing the value of the spectroscopic parameter $F$ - the partition coefficient of the probe TEMPO (Fig. 3), as a function of the concentration of HS: (a) for acids extracted from peat and (b) for model HS. HS extracted from peat, including both HA and FA, slightly stiffened the surface area of the lipid bilayer, as confirmed by the decreasing value of coefficient $F$ in correlation with an increasing concentration of HS. In contrast, FA and HA extracted from model HS interact differently: FA slightly liquefied the surface layer of the membrane, while HA stiffened it (Fig. 3). Figure 4 shows the changes of the spectroscopic parameters - rotational correlation time $\tau$ as a function of the concentration of HAs: a) for acid extracted from peat and $b$ ) the model forms. Both fractions of HS extracted from peat, HA, and FA, increased the fluidity of the central area of the lipid bilayer, as confirmed by the declined value of parameter $\tau$, together with increasing concentrations of HAs. At the same time, HAs had a slightly stronger influence than FAs on the fluidity changes of liposome membranes. In model substances, increased HA concentrations increased the fluidity of the liposome membranes. In contrast, increased concentrations of FA extracted from model substances clearly stiffened the lipid bilayer (Fig. 4).

\section{Conclusions}

1. Fulvic acids (FAs) and humic acids (HAs), regardless of their source, affect the membrane fluidity of liposomes in different ways.

2. Increasing the concentration of HAs extracted from model substances and peat has a similar effect, namely, stiffening the surface of the lipid layer and liquefaction of their middle part. However, when compared to model FAs, the effect of increased concentrations of FAs extracted from peat is the opposite.

3. Fulvic acids, extracted from peat, caused fluidity of the central region of the lipid bilayer, and slightly modified the fluidity of the surface layer (stiffening it). This may indicate that the molecules of FA migrate to the center of the membrane and weaken the bonds between the hydrocarbon chains of phospholipid molecules.

4. Humic acids, extracted from peat, similar to model forms of acids, stiffened the membrane surface area and slightly influenced the hydrophobic interior. This may mean that these compounds are located just below the polar heads of phospholipid molecules. It is possible that HAs, spinning the surface layer, as a consequence cause stiffening.

Acknowledgment. We wish to thank Delia Gosik, native speaker, for proofreading.

\section{References}

1. Schnitzer, M., \& Khan, S. U. (1978). Soil organic matter. Amsterdam: Elsevier. 
2. Pisarek, I. (2003). Characterization of humic substances formed in soil fertilized with sewage sludge and cattle manure. In Humic Substances in Ecosystems 5 (pp. 93-99). Duszniki Zdrój, Poland.

3. Choudhry, G. G. (1984). Humic substances: structural, photophysical, photochemical and free radical aspects and interactions with environmental chemicals. New York: Gordon and Breach Science Publishers.

4. Buffle, J. A. E. (1977). Les substances humiques et leurs interactions avec les ions mineraux. In Conference Proceedings de la Commission d'Hydrologie Appliquee de A.G.H.T.M. (pp. 3-10). l'Universite d'Orsay.

5. Schulten, H. R., Plage, B., \& Schnitzer, M. (1991). A chemical structure for humic substances. Naturwissenschaften, 78, 311-312.

6. Jerzykiewicz, M., Czechowski, F., Jezierski, A., \& Drozd, J. (1999). Influence of ammonia and nitrogen dioxide on free radicals in humic acids derived from composts, soil, peat and brown coal. An EPR study. Humic Subst. Environ., 1(3/4), 21-26.

7. Pisarek, I., Głowacki, M., \& Czernia, M. (2012). The impact of Pleurotus ostreatus on organic matter transformation processes. Water Sci. Technol., 66(12), 2660-2673. DOI: 10.2166/WST.2012.502.

8. Paul, A., Stosser, R., Zehl, A., Zwirnmann, E., Vogt, R., \& Steinberg, C. E. W. (2006). Nature and abundance of organic radicals in natural organic matter: Effect of $\mathrm{pH}$ and irradiation. Environ. Sci. Technol., 40, 5897-5903.

9. Man, D., Słota, R., Mele, G., \& Li, J. (2008). Fluidity of liposome membranes doped with metalloporphyrins: ESR study. Z. Naturforsch. C, 65, 440-444.
10. Polewski, K., Sławińska, D., Sławiński, J., \& Pawlak, A. (2005). The effect of UV and visible light radiation on natural humic acid. EPR spectral and kinetic studies. Geoderma, 126, 291-299.

11. Boniewska-Bernacka, E., Man, D., Słota, R., \& Broda, M. (2011). Effect of tin and lead chlorotriphenyl analogues on selected living cells. J. Biochem. Mol. Toxicol., 25(4), 231-237.

12. Mitrus, S., \& Man, D. (2012). Effect of tin and lead chlorotriphenyl analogues on fruit fly Drosophila hydei and liposomes membrane. J. Biochem. Mol. Toxicol., 26(4), 162-167.

13. Man, D., Podolak, M., \& Engel, G. (2006). The influence of tin compounds on the dynamic properties of liposome membranes: A study using the ESR method. Cell. Mol. Biol. Lett., 11, 56-61.

14. Man, D., Pisarek, I., Braczkowski, M., Pytel, B., \& Olchawa, R. (2014). The impact of humic and fulvic acids on the dynamic properties of liposome membranes: the ESR method. J. Liposome Res., 24(2), 106-112.

15. Soil Taxonomy. (1999). A basic system of soil classification for making and interpreting soil surveys. Soil Survey Staff. USDA-NRCS: US Gort. Printing Office, Washington, DC (Agric. Hand. 436).

16. Man, D., Słota, R., Broda, M. A., Mele, G., \& Li, J. (2011). Metalloporphyrin intercalation in liposome membranes: ESR study. J. Biol. Inorg. Chem., 16(1), 173-181.

17. Shimshick, E. J., \& McConnell, H. M. (1973). Lateral phase separation in phospholipid membranes. Biochemistry, 12, 2351-2360.

18. Hemminga, M. A. (1973). Interpretation of ESR and saturation transfer ESR spectra of spin labeled lipids and membranes. Chem. Phys. Lipids, 32, 323-383. 